\title{
Lysine analogue use during cancer surgery: a survey from a Canadian tertiary care centre
}

\author{
J. Montroy MSc, ${ }^{*}$ B. Hutton $\mathrm{PhD},{ }^{*}$ D.A. Fergusson $\mathrm{PhD}^{* \dagger}{ }^{* \dagger}$ A. Tinmouth MD MSc, ${ }^{*}$ L.T. Lavallée MD MSc, ${ }^{* \dagger}$ \\ I. Cagiannos MD, ${ }^{\ddagger}$ C. Morash MD, ${ }^{\ddagger}$ A. Flaman $\mathrm{PhD}_{1}^{*}$ and R.H. Breau MD MSc${ }^{*+\neq}$
}

\begin{abstract}
Background When used during surgery, antifibrinolytic hemostatic agents such as lysine analogues are effective at reducing blood loss and the need for transfusions. Despite proven efficacy, use of hemostatic agents remains low during some surgeries. Our objective was to explore surgeon opinions about, and use of lysine analogues in, oncologic surgeries at a large tertiary care academic institution.

Methods We administered a survey to surgeons who perform high-transfusion-risk oncologic surgeries at a large academic hospital in Ottawa, Ontario. Design and distribution of the survey followed a modified Dillman method. To ensure that the survey questionnaire was relevant, clear, and concise, we performed informant interviews, cognitive interviews, and pilot-testing. The final survey consisted of 19 questions divided into 3 sections: respondent demographics, use of hemostatic agents, and potential clinical trial opinions.
\end{abstract}

Results Of 28 surgeons, 24 (86\%) participated. When asked to indicate the frequency of lysine analogue use, "never" accounted for $46 \%$ of the responses, and "rarely" ( $<10 \%$ of the time) accounted for $23 \%$ of the responses. Reasons for never using included "unfamiliar with benefits" and "prefer alternatives." Fifteen surgeons (63\%) felt that a trial was needed to demonstrate the efficacy and safety of lysine analogues in their cancer field.

Conclusions Our survey found that lysine analogues are infrequently used during oncologic surgeries at our institution. Many surgeons are unfamiliar with the benefits and side effects of lysine analogues and, alternatively, use topical hemostatic agents. Our results demonstrate that future trials exploring the efficacy and safety of lysine analogues in oncologic surgery are needed.

Key Words Surgery, thrombosis, lysine analogues, tranexamic acid, surveys

Curr Oncol. 2020 December:27(6)e560-e568

www.current-oncology.com

\section{INTRODUCTION}

Bleeding remains one of the most common complications associated with surgery ${ }^{1,2}$. Blood in the surgical field enhances surgical difficulty by obscuring visualization of anatomic structures. Furthermore, excessive blood loss can result in hemodynamic instability, decreased end-organ perfusion, and coagulopathy. Significant blood loss during surgery might necessitate allogenic blood transfusion, which can expose patients to risk of adverse transfusion reactions including infectious and non-infectious events ${ }^{3,4}$. Interventions to reduce surgical blood loss and the need for transfusion are important.

Antifibrinolytic hemostatic agents, such as lysine analogues, have been shown to reduce surgical blood loss and the need for transfusions $s^{5-7}$. These agents are routinely used for cardiac procedures ${ }^{6,8}$ and some orthopedic procedures $^{9,10}$. The efficacy and safety of lysine analogues have also been demonstrated for liver transplantation ${ }^{11}$, total hip arthroplasty ${ }^{12}$, spinal surgery ${ }^{13}$, cardiac surgery ${ }^{6}$, pelvic surger $y^{5}$, pediatric surgeries ${ }^{14}$, and many others ${ }^{15,16}$. Lysine analogues are used at the discretion of the attending surgeon and anesthesiologist, but considering the evidence supporting their use, coupled with their relatively low cost, it is surprising that their adoption in certain areas of surgery has been low ${ }^{17,18}$.

Oncologic surgery is a field that has been particularly slow to take up lysine analogues ${ }^{17,19}$. A recent systematic review and meta-analysis found that only 11 studies of lysine analogues have been performed in oncology patients. In 
addition, only 9 of those studies were conducted in a surgical setting, and a considerable degree of heterogeneity was observed with respect to cancer type and surgical procedures performed $^{19}$. Patients with cancer are often anemic before surgery because of receipt of neoadjuvant chemotherapy or because of bleeding from a tumour, and the procedures that those patients require are often associated with substantial blood loss ${ }^{20}$. The impact of lysine analogue use in those patients could therefore be substantial. Conversely, cancer is a risk factor for thrombosis, and therefore any therapy that might theoretically induce clotting (that is, lysine analogues) should be evaluated for safety. The objective of the present study was to survey surgeons concerning their opinions about lysine analogue use during high-transfusionrisk cancer surgery. We also sought to determine whether surgeons believe that further trials are required before lysine analogues should be used in cancer patients.

\section{METHODS}

\section{Study Population and Recruitment}

The survey was intended and developed for surgeons at The Ottawa Hospital who perform hepatectomy, Whipple or pancreatectomy, rectal resection, radical colectomy, radical cystectomy, radical nephrectomy, radical hysterectomy, or large abdominal tumour resection. The Ottawa Hospital is a large tertiary academic centre serving more than 1.2 million residents in the city of Ottawa and surrounding areas of Eastern Ontario. Procedures were selected because they are both high-risk for transfusion and commonly performed at our institution ${ }^{21}$. All the procedures evaluated used an open approach, as opposed to laparoscopic. Contact was made with the head of each clinical department to obtain a complete list of surgeons within their department who perform the listed procedures. Identified surgeons were invited by e-mail to participate in the online survey, which made use of Google Forms (Google, Mountain View, CA, U.S.A.). Reminders were sent by e-mail to non-responders at 2-week intervals for 4 weeks in a modified Dillman method ${ }^{22}$. No financial or other incentive was offered for completion of the survey. Local research ethics board approval was obtained before survey dissemination, which occurred in May 2017.

\section{Survey Design and Distribution}

The survey was developed by clinicians and researchers with expertise in the fields of surgery, anesthesia, blood transfusion, clinical trials, and survey methodology. Key informant interviews, cognitive interviews, and pilot-testing were performed to achieve a survey that was relevant, clear, and concise. The final survey consisted of 19 questions divided into 3 sections: respondent demographics, hemostatic agent use, and clinical trial opinions (Table I).

Demographic questions included surgical procedures performed, years in practice, and geographic location of fellowship training. Participants were asked to estimate the number of procedures performed per year, and the proportion of patients who required a blood transfusion. For each procedure, surgeons were asked to estimate the proportion of their patients who received lysine analogues, which lysine analogue was used, and how the drug was administered. Surgeons who reported lysine analogue use were then asked to indicate the timing of lysine analogue administration. Surgeons were also asked about their reasons for not using lysine analogues and asked to indicate other techniques used to limit blood loss and the need for transfusion. Lastly, surgeons were asked if they believed that a clinical trial of lysine analogues was necessary before those agents could be used. When a "no" answer was given, the surgeon was asked to indicate the reason for that response. If applicable, respondents were also asked to rank potential trial endpoints in order of importance.

\section{Data Analysis}

Survey responses were imported into a Microsoft Excel spreadsheet for analysis (version 15.0: Microsoft Corporation, Redmond, WA, U.S.A.).

Data from questions with categorical outcomes were summarized using proportions. Data from free-text questions were collated. Univariable regression was used to estimate odds ratios for lysine analogue use by surgical specialty, completion of a fellowship, years since fellowship completion, and location of surgical training. For the purpose of the analysis, a dichotomous variable " $<10 \%$ use" was created, grouping responses of "never" and "rarely"; "sometimes," "often," and "always" were then combined. That dichotomization represents a simple and feasible approach to identifying lysine analogue users and nonusers. All analyses were performed using the SAS software application (version 9.4: SAS Institute, Cary, NC, U.S.A.).

\section{RESULTS}

\section{Demographics and Response Rate}

We identified 28 surgeons at our institution who had performed at least 1 of the eligible high-transfusion-risk cancer procedures. All 28 were invited to participate in the survey. Of those 28 surgeons, 24 (86\%) completed the survey. Stratified by surgical subspecialty, response rates were 11 of 13 in general surgery (85\%), 9 of 10 in urologic surgery (90\%), and 4 of $5(80 \%)$ in gynecologic surgery. Table II summarizes the characteristics of the responding surgeons. Surgeon experience showed a wide distribution, ranging from less than 5 years in practice to more than 20 years. Most responding surgeons (58\%) were trained in Canada; others had received training in the United States (29\%), Australia (8\%), and the United Kingdom (4\%). Of the 24 responding surgeons (8\%), 2 did not receive fellowship subspecialty training.

\section{Estimated Proportion of Patients Transfused}

Surgeons were asked to estimate the proportion of their surgical patients requiring perioperative blood transfusion. Self-reported transfusion rates were low, with $94 \%$ of respondents reporting a transfusion proportion of less than $20 \%$. The procedure for which surgeons reported a transfusion rate greater than $30 \%$ was radical cystectomy.

\section{Lysine Analogue Use}

The only lysine analogue used by respondents was tranexamic acid. Lysine analogues were reported as "never used" in roughly $40 \%$ of procedures and "rarely used" $(<10 \%$ 
TABLE I Final survey administered to surgeons

Lysine analogue use in cancer surgery

Dear survey respondent,

You are invited to participate in a short survey on the use of lysine analogues during surgery. Lysine analogues are hemostatic agents shown to be effective at reducing blood loss and transfusions. You were selected as a possible participant in this study because you are a surgeon at The Ottawa Hospital.

The survey is designed to explore current knowledge and use of lysine analogues, as well as potential reasons why you may not use this drug. The survey will take less than 10 minutes to complete and its completion implies your consent. No benefits accrue to you for answering the survey, but your responses will be valuable in assisting with the design of a potential clinical trial.

All information obtained will remain confidential and will not be disclosed except in the aggregate. If you decide to participate, you are free to discontinue participation at any time without prejudice.

If you have any questions, please ask. If you have additional questions later, contact Joshua Montroy or Rodney Breau.

Thank you for your time.

Sincerely,

Dr. Rodney Breau

Dr. Dean Fergusson

Joshua Montroy

\section{Section I - Demographics}

1. Do you perform any of the following surgeries? (check all that apply)

$\square$ Cystectomy $\quad \square$ Open nephrectomy $\quad \square$ Hepatectomy $\square$ Whipple procedure $\square$ Open splenectomy

$\square$ Large abdominal tumour resection $\quad \square$ Open colectomy $\quad \square$ Pancreatectomy $\quad \square$ Open total rectal resection

$\square$ Open radical or debulking hysterectomy

2. Indicate the number of years since you completed residency and fellowship
$\square>5$ years
$\square$ 5-10 years
$\square$ 11-15 years
$\square$ 16-20 years
$\square>20$ years

3. Did you receive fellowship subspecialty training?

$\square$ No $\square$ Yes

4. Where did you complete your most recent surgical training?

$\square$ Canada $\quad \square$ U.S.A. $\quad \square$ U.K. $\quad \square$ Other, please specify:

5. $\quad$ For each surgery, approximately how many of the listed surgeries did you personally perform per year over the last five years?

\begin{tabular}{|c|c|c|c|c|c|c|}
\hline & $<10$ & $10-24$ & $25-49$ & $50-75$ & $>75$ & Not performed \\
\hline Radical cystectomy & $\square$ & $\square$ & $\square$ & $\square$ & $\square$ & $\square$ \\
\hline Hepatectomy & $\square$ & $\square$ & $\square$ & $\square$ & $\square$ & $\square$ \\
\hline Pancreas resection & $\square$ & $\square$ & $\square$ & $\square$ & $\square$ & $\square$ \\
\hline Open splenectomy & $\square$ & $\square$ & $\square$ & $\square$ & $\square$ & $\square$ \\
\hline Open radical or debulking hysterectomy & $\square$ & $\square$ & $\square$ & $\square$ & $\square$ & $\square$ \\
\hline Open nephrectomy & $\square$ & $\square$ & $\square$ & $\square$ & $\square$ & $\square$ \\
\hline Large abdominal tumour resection & $\square$ & $\square$ & $\square$ & $\square$ & $\square$ & $\square$ \\
\hline Open total colectomy & $\square$ & $\square$ & $\square$ & $\square$ & $\square$ & $\square$ \\
\hline Whipple procedure & $\square$ & $\square$ & $\square$ & $\square$ & $\square$ & $\square$ \\
\hline Open total rectal resection & $\square$ & $\square$ & $\square$ & $\square$ & $\square$ & $\square$ \\
\hline
\end{tabular}

6. Estimate the percentage of your surgical patients (for the listed surgeries) who require a perioperative blood transfusion.

\begin{tabular}{|c|c|c|c|c|c|}
\hline & $<10 \%$ & $10 \%-19.9 \%$ & $20 \%-29.9 \%$ & $30 \%-49.9 \%$ & $>50 \%$ \\
\hline Radical cystectomy & $\square$ & $\square$ & $\square$ & $\square$ & $\square$ \\
\hline Hepatectomy & $\square$ & $\square$ & $\square$ & $\square$ & $\square$ \\
\hline Pancreas resection & $\square$ & $\square$ & $\square$ & $\square$ & $\square$ \\
\hline Open splenectomy & $\square$ & $\square$ & $\square$ & $\square$ & $\square$ \\
\hline Open radical or debulking hysterectomy & $\square$ & $\square$ & $\square$ & $\square$ & $\square$ \\
\hline Open nephrectomy & $\square$ & $\square$ & $\square$ & $\square$ & $\square$ \\
\hline Large abdominal tumour resection & $\square$ & $\square$ & $\square$ & $\square$ & $\square$ \\
\hline Open total colectomy & $\square$ & $\square$ & $\square$ & $\square$ & $\square$ \\
\hline Whipple procedure & $\square$ & $\square$ & $\square$ & $\square$ & $\square$ \\
\hline Open total rectal resection & $\square$ & $\square$ & $\square$ & $\square$ & $\square$ \\
\hline
\end{tabular}




\section{Section I - Demographics continued}

7. Estimate the percentage of your surgical patients (for the listed surgeries) who require a postoperative blood transfusion (from surgery to 30 days postoperative)

\begin{tabular}{|c|c|c|c|c|c|}
\hline & $<10 \%$ & $10 \%-19.9 \%$ & $20 \%-29.9 \%$ & $30 \%-49.9 \%$ & $>50 \%$ \\
\hline Radical cystectomy & $\square$ & $\square$ & $\square$ & $\square$ & $\square$ \\
\hline Hepatectomy & $\square$ & $\square$ & $\square$ & $\square$ & $\square$ \\
\hline Pancreas resection & $\square$ & $\square$ & $\square$ & $\square$ & $\square$ \\
\hline Open splenectomy & $\square$ & $\square$ & $\square$ & $\square$ & $\square$ \\
\hline Open radical or debulking hysterectomy & $\square$ & $\square$ & $\square$ & $\square$ & $\square$ \\
\hline Open nephrectomy & $\square$ & $\square$ & $\square$ & $\square$ & $\square$ \\
\hline Large abdominal tumour resection & $\square$ & $\square$ & $\square$ & $\square$ & $\square$ \\
\hline Open total colectomy & $\square$ & $\square$ & $\square$ & $\square$ & $\square$ \\
\hline Whipple procedure & $\square$ & $\square$ & $\square$ & $\square$ & $\square$ \\
\hline Open total rectal resection & $\square$ & $\square$ & $\square$ & $\square$ & $\square$ \\
\hline
\end{tabular}

\section{Section II - Hemostatic agent use}

8. Are you aware of the hemostatic agents known as lysine analogues? (e.g., Cyklokaprona, tranexamic acid, aminocaproic acid, Amicarb) $\square$ Yes $\square$ No

9. How often do you administer lysine analogues during any of the listed surgeries? (check all that apply)

\begin{tabular}{|c|c|c|c|c|c|c|}
\hline & Never & $\begin{array}{c}\text { Rarely } \\
(<10 \%)\end{array}$ & $\begin{array}{c}\text { Sometimes } \\
(\mathbf{1 0} \%-\mathbf{4 0} \%)\end{array}$ & $\begin{array}{c}\text { Often } \\
(40 \%-75 \%) \\
\end{array}$ & $\begin{array}{l}\text { Always } \\
(>75 \%)\end{array}$ & Never \\
\hline Radical cystectomy & $\square$ & $\square$ & $\square$ & $\square$ & $\square$ & $\square$ \\
\hline Hepatectomy & $\square$ & $\square$ & $\square$ & $\square$ & $\square$ & $\square$ \\
\hline Pancreas resection & $\square$ & $\square$ & $\square$ & $\square$ & $\square$ & $\square$ \\
\hline Open splenectomy & $\square$ & $\square$ & $\square$ & $\square$ & $\square$ & $\square$ \\
\hline Open radical or debulking hysterectomy & $\square$ & $\square$ & $\square$ & $\square$ & $\square$ & $\square$ \\
\hline Open nephrectomy & $\square$ & $\square$ & $\square$ & $\square$ & $\square$ & $\square$ \\
\hline Large abdominal tumour resection & $\square$ & $\square$ & $\square$ & $\square$ & $\square$ & $\square$ \\
\hline Open total colectomy & $\square$ & $\square$ & $\square$ & $\square$ & $\square$ & $\square$ \\
\hline Whipple procedure & $\square$ & $\square$ & $\square$ & $\square$ & $\square$ & $\square$ \\
\hline Open total rectal resection & $\square$ & $\square$ & $\square$ & $\square$ & $\square$ & $\square$ \\
\hline
\end{tabular}

10. Which of the following lysine analogues do you administer to patients? (check all that apply)

$\square$ Tranexamic acid (Cyklokapron $\left.{ }^{\mathrm{a}}\right) \quad \square$ Aminocaproic acid (Amicar $\left.{ }^{\mathrm{b}}\right) \quad \square$ None

11. How do you typically administer lysine analogues to your patients? (check all that apply)

$\square$ As preoperative prophylaxis

$\square$ As intraoperative prophylaxis

$\square$ As postoperative prophylaxis

$\square$ Combination of above 3 techniques

$\square$ Intraoperatively if needed

$\square$ Not applicable

12. If you do not administer lysine analogues during the procedures listed above, what are the reasons? (check all that apply):

$\square$ Not applicable (I do administer lysine analogues)

$\square$ Unfamiliar with benefits $\quad \square$ Unfamiliar with side effects $\quad \square$ Lack of availability

$\square$ Unfamiliar with method of use $\quad \square$ Cost $\quad \square$ Safety concerns

$\square$ Prefer alternatives to prevent blood loss

13. What other techniques do you use to limit surgical blood loss or transfusion in your patients?

$\square$ Topical hemostatic agents (e.g., Surgicelc, Gelfoama, ${ }^{\mathrm{a}}$, Tisseeld ${ }^{\mathrm{d}}$, Floseald ${ }^{\mathrm{d}}$ )

$\square$ Autologous blood recovery (Cell Savere) $\quad \square$ Autologous blood banking

$\square$ Acute normovolemic hemodilution $\square$ Preoperative iron therapy

$\square$ Other intravenous medications (i.e., factor VII)

$\square$ Restrictive transfusion triggers (intraoperatively and postoperatively) $\quad \square$ Not applicable 
TABLE I Continued

\section{Section III - Potential Clinical Trial}

14. Do you feel as if a trial is needed to demonstrate the efficacy of lysine analogues? If not, why?

$\square$ Yes $\square$ No:

15. Do you feel as if a trial is needed to demonstrate the safety of lysine analogues? If not, why?

$\square$ Yes $\square$ No:

16. Consider a randomized controlled trial of lysine analogue administration to minimize blood loss and transfusion. For which procedure(s) would you be willing to enrol patients?

$\square$ I am not interested in a trial of this nature $\quad$ I would be willing to enrol all patients in a trial

$\square$ Cystectomy $\quad \square$ Open radical or debulking hysterectomy $\quad \square$ Hepatectomy

$\square$ Open nephrectomy $\square$ Whipple procedure $\square$ Open splenectomy $\quad \square$ Open colectomy

$\square$ Large abdominal tumour resection $\quad \square$ Pancreatectomy $\quad \square$ Open total rectal resection

17. Consider a randomized controlled trial of lysine analogue administration in the above listed surgeries. Can you indicate what you would consider to be the most clinically relevant primary endpoint by ranking these endpoints ( 1 being most important, 3 being least important):

$\square$ Proportion of patients transfused

$\square$ Total units transfused

$\square$ Incidence of thromboembolic events (DVT and PE)

18. To consider incorporating lysine analogues into routine practice, they would need to reduce the relative risk of transfusion risk what percentage?
$\square 20 \%$ (e.g., $30 \%$ risk to $26 \%$ risk)
$\square 50 \%$ (e.g., $30 \%$ risk to $15 \%$ risk)
$\square 30 \%$ (e.g., $30 \%$ risk to $21 \%$ risk)
$\square 40 \%$ (e.g., $30 \%$ risk to $18 \%$ risk)
$\square>50 \%$

19. If lysine analogues were to reduce the red blood cell transfusion rate by $33 \%$ (for example from $30 \%$ down to $20 \%$ ), what relative increase in risk of thromboembolic events (confirmed DVT and PE) would be acceptable?
$\square<20 \%$ (e.g., $5 \%$ risk to $<6 \%$ risk)
$\square 20 \%$ (e.g., $5 \%$ risk to $6 \%$ risk)
$\square 30 \%$ (e.g., $5 \%$ risk to $6.5 \%$ risk)
$\square 40 \%$ (e.g., $5 \%$ risk to $7 \%$ risk)

afizer, New York, NY, U.S.A.

b Clover Pharmaceuticals, Lake Forest, IL, U.S.A.

Ethicon, Cincinnati, OH, U.S.A.

d Baxter International, Deerfield, IL, U.S.A.

e Haemonetics Corporation, Boston, MA, U.S.A.

DVT = deep-vein thrombosis; $\mathrm{PE}=$ pulmonary embolism.

TABLE II Demographic characteristics of the survey respondents

\begin{tabular}{|c|c|c|c|c|}
\hline \multirow[t]{2}{*}{ Characteristic } & \multicolumn{4}{|c|}{ Surgical specialty $[n(\%)]$} \\
\hline & General & Urologic & Gynecologic & Overall \\
\hline Invitations to participate & 13 & 10 & 5 & 28 \\
\hline Respondents & 11 & 9 & 4 & 24 \\
\hline $\begin{array}{l}\text { Procedures performed } \\
\text { Hepatectomy } \\
\text { Large abdominal tumour resection } \\
\text { Pancreatectomy } \\
\text { Whipple procedure } \\
\text { Open colectomy } \\
\text { Open rectal resection } \\
\text { Radical cystectomy } \\
\text { Open nephrectomy } \\
\text { Open radical hysterectomy }\end{array}$ & $\begin{array}{c}3 \\
6 \\
5 \\
3 \\
11 \\
7\end{array}$ & $\begin{array}{l}3 \\
8\end{array}$ & 4 & \\
\hline $\begin{array}{l}\text { Time since training completion } \\
\quad<5 \text { Years } \\
5-10 \text { Years } \\
11-15 \text { Years } \\
16-20 \text { Years } \\
>20 \text { Years }\end{array}$ & $\begin{array}{c}5(45.5) \\
3(27.3) \\
1(9.1) \\
0 \\
2(18.2)\end{array}$ & $\begin{array}{c}3(33.3) \\
2(22.2) \\
2(22.2) \\
0 \\
2(22.2)\end{array}$ & $\begin{array}{c}0 \\
0 \\
0 \\
2(50) \\
2(50)\end{array}$ & $\begin{array}{c}8(33.3) \\
5(20.8) \\
3(12.5) \\
2(8.3) \\
6(25)\end{array}$ \\
\hline $\begin{array}{l}\text { Location of surgical training } \\
\text { Canada } \\
\text { United States } \\
\text { Other }\end{array}$ & $\begin{array}{l}4(36.4) \\
4(36.4) \\
3(27.3)\end{array}$ & $\begin{array}{c}6(66.7) \\
3(33.3) \\
0\end{array}$ & $\begin{array}{c}4(100) \\
0 \\
0\end{array}$ & $\begin{array}{l}14(58.3) \\
7(29.2) \\
3(12.5)\end{array}$ \\
\hline $\begin{array}{l}\text { Fellowship training } \\
\text { Yes } \\
\text { No }\end{array}$ & $\begin{array}{c}11(100) \\
0\end{array}$ & $\begin{array}{c}8(88.9) \\
1(11.1)\end{array}$ & $\begin{array}{l}3(75) \\
1(25)\end{array}$ & $\begin{array}{r}22(91.7) \\
2(8.3)\end{array}$ \\
\hline
\end{tabular}


of the time) in another $20 \%$ of procedures (Table III). Procedures in which at least 1 surgeon used lysine analogues "often" or "always" included hepatectomy, large abdominal tumour resection, pancreatectomy, and radical cystectomy. The timing of drug administration was similar for surgeons and procedures, with 11 respondents $(61.1 \%)$ reporting reactively using a lysine analogue when significant bleeding was encountered (intraoperatively, if needed) and 7 respondents (38.9\%) reporting use of a lysine analogue prophylactically (Table IV).

TABLE III Lysine analogue use by responding surgeons

\begin{tabular}{|c|c|c|c|c|c|c|}
\hline \multirow[t]{2}{*}{ Procedure } & \multirow[t]{2}{*}{$\underset{\text { (n) }}{\text { Surgeons }}$} & \multicolumn{5}{|c|}{$\begin{array}{c}\text { Frequency of lysine analogue use } \\
\text { [n (\%) of surgeons who perform the associated procedure] }\end{array}$} \\
\hline & & Never & $\begin{array}{c}\text { Rarely } \\
(<10 \%)\end{array}$ & $\begin{array}{c}\text { Sometimes } \\
(\mathbf{1 0} \%-\mathbf{4 0} \%)\end{array}$ & $\begin{array}{c}\text { Often } \\
(40 \%-75 \%)\end{array}$ & $\begin{array}{l}\text { Always } \\
(>75 \%)\end{array}$ \\
\hline Hepatectomy & 3 & 0 & 0 & $1(33.3)$ & $1(33.3)$ & $1(33.3)$ \\
\hline Large abdominal tumour resection ${ }^{\mathrm{a}}$ & 6 & $1(20)$ & $1(20)$ & $1(20)$ & $2(40)$ & 0 \\
\hline Pancreatectomy & 5 & 0 & $2(40)$ & $1(20)$ & $2(40)$ & 0 \\
\hline Whipple procedure & 3 & 0 & $2(66.7)$ & $1(33.3)$ & 0 & 0 \\
\hline Open colectomy ${ }^{\mathrm{a}}$ & 11 & $7(70)$ & $1(10)$ & $2(20)$ & 0 & 0 \\
\hline Open rectal resection & 7 & $5(71.4)$ & $1(14.3)$ & $1(14.3)$ & 0 & 0 \\
\hline Radical cystectomy & 3 & 0 & 0 & $2(66.7)$ & $1(33.3)$ & 0 \\
\hline Open nephrectomy & 8 & $5(62.5)$ & $2(25.0)$ & $1(12.5)$ & 0 & 0 \\
\hline Open hysterectomy & 4 & $2(50.0)$ & $2(50.0)$ & 0 & 0 & 0 \\
\hline
\end{tabular}

a One surgeon who performs this procedure did not answer this question.

TABLE IV Patterns of lysine analogue use by survey respondents

\begin{tabular}{|c|c|c|c|c|}
\hline \multirow[t]{2}{*}{ Variable } & \multicolumn{4}{|c|}{ Surgical specialty [n (\%) of respondents] } \\
\hline & General & Urologic & Gynecologic & Overall \\
\hline \multicolumn{5}{|l|}{ Timing of lysine analogue administration } \\
\hline A. Intraoperatively, if needed & $6(54.5)$ & $4(80)$ & $1(50)$ & $11(61.1)$ \\
\hline B. Intraoperative prophylaxis & $4(36.4)$ & $1(20)$ & $1(50)$ & $6(33.3)$ \\
\hline C. Postoperative prophylaxis & $1(9.1)$ & 0 & 0 & $1(5.6)$ \\
\hline D. Preoperative prophylaxis & 0 & 0 & 0 & 0 \\
\hline Combination of B-D & 0 & 0 & 0 & 0 \\
\hline \multicolumn{5}{|l|}{ Reasons for non-use } \\
\hline Unfamiliar with benefits & $2(33.3)$ & $1(33.3)$ & 0 & $3(30.0)$ \\
\hline Prefer alternatives & $1(16.7)$ & $2(66.7)$ & 0 & $3(30.0)$ \\
\hline Unfamiliar with side effects & $1(16.7)$ & 0 & 0 & $1(10.0)$ \\
\hline Safety concerns & $1(16.7)$ & 0 & 0 & $1(10.0)$ \\
\hline Unfamiliar with method of use & 0 & 0 & $1(100)$ & $1(10.0)$ \\
\hline Cost & $1(16.7)$ & 0 & 0 & $1(10.0)$ \\
\hline Lack of availability & 0 & 0 & 0 & 0 \\
\hline \multicolumn{5}{|c|}{ Other blood loss or transfusion prevention techniques used } \\
\hline Topical hemostatic agents & $11(100)$ & $8(100)$ & $4(100)$ & $23(95.8)$ \\
\hline Restrictive transfusion triggers & $7(63.6)$ & 0 & $1(25)$ & $8(33.3)$ \\
\hline Preoperative iron therapy & $5(45.5)$ & 0 & $1(25)$ & $6(25)$ \\
\hline Acute normovolemic hemodilution & $3(27.3)$ & 0 & 0 & $3(12.5)$ \\
\hline Fibrinogen concentrate & $1(9.1)$ & 0 & 0 & $1(4.2)$ \\
\hline Autologous blood recovery (Cell Savera) & 0 & 0 & $1(25)$ & $1(4.2)$ \\
\hline Other intravenous medications (factor VII) & $1(9.1)$ & 0 & 0 & $1(4.2)$ \\
\hline Autologous blood banking & $1(9.1)$ & 0 & 0 & $1(4.2)$ \\
\hline Topical lysine analogues & 0 & 0 & 0 & 0 \\
\hline
\end{tabular}

a Haemonetics Corporation, Boston, MA, U.S.A. 
A number of reasons were provided for not using lysine analogues (Table IV). The most common reason was that surgeons were "unfamiliar with benefits" $(n=3,30 \%)$ or "preferred alternatives" ( $n=3,30 \%)$. The most popular alternative preferred approach to prevent blood loss and the need for transfusion was topical hemostatic agents $(n=23$, 95.8\%; Table IV). Other common blood loss and transfusionprevention techniques included restrictive transfusion triggers $(n=7,33.3 \%)$ and preoperative iron therapy $(n=$ $6,25 \%)$. In our univariable analysis, surgeons who trained in Canada, compared with those trained outside Canada,

TABLE V Univariable analysis of lysine analogue use, by surgical characteristics

\begin{tabular}{|c|c|c|c|}
\hline Variable & Comparator & OR & $95 \% \mathrm{Cl}$ \\
\hline \multicolumn{4}{|l|}{ Surgical specialty } \\
\hline Urology & Gynecology & 2.04 & 0.26 to 9.8 \\
\hline General surgery & Gynecology & 3.51 & 0.49 to 10.1 \\
\hline Urology & General surgery & 0.62 & 0.06 to 5.08 \\
\hline \multicolumn{4}{|c|}{ Years since fellowship } \\
\hline$\geq 10$ Years & $<10$ Years & 0.60 & 0.11 to 3.40 \\
\hline \multicolumn{4}{|l|}{ Location of training } \\
\hline Canada & Outside Canada & 0.11 & 0.02 to 0.79 \\
\hline \multicolumn{4}{|c|}{ Completion of fellowship } \\
\hline Yes & No & 1.25 & 0.14 to 12.5 \\
\hline
\end{tabular}

$\mathrm{OR}=$ odds ratio; $\mathrm{Cl}=$ confidence interval. were less likely to administer lysine analogues (Table v). No other variable tested was associated with lysine analogue use, although the analysis was underpowered, given the limited sample size.

\section{Opinions About the Need for a Lysine Analogue Clinical Trial}

Of the responding surgeons, 18 (75\%) indicated that they believe a trial is needed to demonstrate the efficacy of lysine analogues in their field (Table VI), and 4 (16.7\%) indicated that they do not believe such a trial is needed. The latter 4 indicated that they already believe that the efficacy of lysine analogues has been proved. Fourteen surgeons (58.3\%) felt that a trial was needed to demonstrate the safety of lysine analogues in their respective fields. Because of a belief that the drug has already been proven safe, 8 surgeons (33.3\%) indicated that they do not believe a trial is necessary to demonstrate the safety of lysine analogues in their respective fields.

When asked to rank their preferred primary endpoints in a clinical trial, 20 surgeons $(83.3 \%)$ responded that the most clinically relevant primary outcome of such a trial would be the proportion of patients transfused, $2(8.3 \%)$ indicated that the incidence of thromboembolic events (deep-vein thrombosis and pulmonary embolism) would be the most clinically relevant primary endpoint, and 2 (8.3\%) thought that the total number of units transfused would be the most clinically relevant primary endpoint (Table VI).

TABLE VI Clinical trial interest among survey respondents

\begin{tabular}{|c|c|c|c|c|}
\hline \multirow[t]{2}{*}{ Question } & \multicolumn{4}{|c|}{ Surgical specialty [n (\%) respondents] } \\
\hline & General & Urologic & Gynecologic & Overall \\
\hline \multicolumn{5}{|l|}{ Trial needed to demonstrate efficacy? } \\
\hline Yes & $6(54.5)$ & $9(100)$ & $3(75.0)$ & $18(75.0)$ \\
\hline No & $3(27.2)$ & 0 & $1(25.0)$ & $4(16.7)$ \\
\hline Unsure & $2(18.2)$ & 0 & 0 & $2(8.3)$ \\
\hline \multicolumn{5}{|l|}{ Trial needed to demonstrate safety? } \\
\hline Yes & $4(36.4)$ & $7(77.8)$ & $3(75.0)$ & $14(58.3)$ \\
\hline No & $5(45.5)$ & $2(22.2)$ & $1(25.0)$ & $8(33.3)$ \\
\hline Unsure & $2(18.2)$ & 0 & 0 & $2(8.3)$ \\
\hline \multicolumn{5}{|l|}{ Desired primary outcome } \\
\hline Proportion of patients transfused & $11(100)$ & $6(66.7)$ & $3(75.0)$ & $20(83.3)$ \\
\hline Incidence of thromboembolic events & 0 & $2(22.2)$ & 0 & $2(8.3)$ \\
\hline Total blood units transfused & 0 & $1(11.1)$ & $1(25.0)$ & $2(8.3)$ \\
\hline \multicolumn{5}{|l|}{ Desired efficacy ${ }^{a}$} \\
\hline $20 \%$ & 9 (81.8) & $5(55.6)$ & $3(75.0)$ & $17(70.8)$ \\
\hline $30 \%$ & $1(9.1)$ & $2(22.2)$ & $1(25.0)$ & $4(16.7)$ \\
\hline $40 \%$ & 0 & $2(22.2)$ & 0 & $2(8.3)$ \\
\hline $50 \%$ & $1(9.1)$ & 0 & 0 & $1(4.2)$ \\
\hline$>50 \%$ & 0 & 0 & 0 & 0 \\
\hline \multicolumn{5}{|l|}{ Tolerable risk ${ }^{b}$} \\
\hline$<20 \%$ & 9 (81.8) & 8 (88.9) & $4(100)$ & $21(87.5)$ \\
\hline $20 \%$ & $2(18.2)$ & $1(11.1)$ & 0 & $3(12.5)$ \\
\hline $30 \%$ & 0 & 0 & 0 & 0 \\
\hline $40 \%$ & 0 & 0 & 0 & 0 \\
\hline
\end{tabular}

a Relative reduction in transfusion risk.

b Relative increase in risk of venous thromboembolism. 
Most of the responding surgeons (70.1\%) indicated that a minimum $20 \%$ reduction in the relative risk for transfusion would be enough to routinely use a lysine analogue during surgery. A greater proportion (87.5\%) responded that an increase in the relative risk of thromboembolic events of less than $20 \%$ would be tolerable (Table VI).

\section{DISCUSSION}

Despite improvements in surgical technique and hemostatic drug development, surgical blood loss and a related need for transfusions commonly occur ${ }^{3,4}$. In 1996, Canadian hospitals were surveyed about lysine analogue use across surgical disciplines ${ }^{23}$. Lysine analogues were rarely used, except in cardiac procedures. Since then, evidence has accumulated supporting the use of lysine analogues to minimize blood loss during surgery. Patients with cancer, who frequently require blood transfusion because of both preoperative anemia and the complexity of the surgical procedures required to completely remove tumours ${ }^{20}$, might benefit from the perioperative use of lysine analogues. We therefore developed a survey to examine whether and how surgeons who perform high-transfusion-risk oncologic procedures at our institution use lysine analogues.

Our survey revealed that lysine analogue use is inconsistent and infrequent during cancer surgery. Of surgeons who perform high-transfusion-risk oncologic procedures, roughly two thirds $(64.6 \%)$ "never" or "rarely" used lysine analogues. In the instances in which lysine analogues were used, discrepancies in their administration were revealed: 11 surgeons administered lysine analogues as a reactionary measure in the presence of major bleeding; only 7 used lysine analogues as a prophylactic measure. That variability in lysine analogue use could be anticipated, given that guidelines establishing the timing and dose of lysine analogues do not exist ${ }^{24,25}$. Other reasons for non-use were lack of familiarity with the benefits and risks of the drugs.

Although the absence of a protocol might hinder some surgeons from using lysine analogues, others might hesitate because good evidence for the use of those agents in their field of surgery does not exist. Randomized trials have proven the efficacy and safety of lysine analogues in many specialties - indeed, lysine analogues are routinely used for bleeding prophylaxis in cardiac and orthopedic surgery ${ }^{6,10,26}$. However, evidence supporting lysine analogue use in patients with cancer is more limited ${ }^{19}$. Furthermore, oncology surgeons might adopt the use of lysine analogues more readily if they had confidence in the safety of those drugs. Lysine analogues have been associated with an increased risk of thrombosis, and concerns about that risk will persist until the relationship is examined in a properly designed and powered clinical trial. Those safety concerns are further exacerbated in the oncology population because, compared with the general population, patients with a malignant disease have an increased risk of thrombosis ${ }^{24,25}$. A recent systematic review of lysine analogues in patients with cancer identified only 7 trials evaluating transfusion outcomes and 9 evaluating safety [venous thromboembolism (VTE)] outcomes ${ }^{19}$. A pooled analyses of those studies lacked the precision to make definitive conclusions about the risk of VTE associated with lysine analogues in patients with cancer (relative risk: 0.60; 95\% confidence interval: 0.28 to 1.3 ).

Most surgeons at our institution indicated that new trials are needed to establish the benefit and safety of lysine analogues during cancer surgery. Although most responding surgeons felt that the most clinically relevant endpoint of a trial of lysine analogue use would be efficacy, 2 surgeons indicated that the incidence of thromboembolic events would be of greater importance. Surgeons in our survey were asked to indicate, given the blood loss and transfusion benefits of lysine analogues, what increase in the risk of VTE they could tolerate. Twenty-one surgeons indicated that they would accept only a relative increase of less than $20 \%$ in VTE risk. That level presents a major challenge in designing a trial with VTE as the primary endpoint, given that an increased risk of less than $20 \%$ over the low baseline incidence of VTE $(\leq 5 \%)$ represents an absolute increase of less than $1 \%$, making it nearly impossible to properly power a trial. Safety concerns with respect to the consequences of VTE remain a barrier for the implementation of routine lysine analogue use in the oncology population.

Contrasting with the hesitation on the part of our respondents to adopt lysine analogues to minimize bleeding, almost all responding surgeons $(n=23)$ indicated that they use topical hemostatic agents such as Surgicel (Ethicon, Cincinnati, OH, U.S.A.) and Gelfoam (Pfizer, New York, NY, U.S.A.) to prevent blood loss. Those agents have been heavily marketed, but few high-powered randomized clinical trials have been conducted to demonstrate their efficacy ${ }^{27}$, especially compared with the many trials supporting the use of lysine analogues. Other popular methods for reduction of blood loss identified in our survey included restrictive transfusion triggers, autologous blood recovery, acute normovolemic hemodilution, and preoperative iron therapy. All have been shown to be effective techniques ${ }^{28-31}$, albeit with some limitations. For example, a systematic review of seventy-five trials demonstrated a higher risk of infection associated with preoperative iron therapy ${ }^{32}$. Surgeons who treat oncology patients while using autologous blood recovery should be alerted to the theoretical possibility and increased risk of cancer dissemination ${ }^{33,34}$. A recent systematic review and meta-analysis of acute normovolemic hemodilution demonstrated that, although results suggest that it is effective in reducing the need for transfusion, significant heterogeneity and publication bias raised serious concerns about the true efficacy of the technique ${ }^{31}$.

Our study has limitations. We did not survey anesthesiologists, who are often responsible for ordering the use of lysine analogues, and we might therefore be underestimating lysine analogue use at our institution. It would be valuable to survey that group as well. Because we communicated with the head of the department to identify surgeons in each specialty for contact, it is unlikely that we omitted possible respondents. Our chosen method of contact, coupled with our reasonable response rate (86\%), allows us to conclude that our results can be generalized to the surveyed specialties throughout our institution. However, because a standard protocol for lysine analogue use does not exist, our results might not translate to other institutions-although a survey of urologic oncologists ${ }^{17}$ and hepatic surgeons ${ }^{18}$ across Canada suggested similar rates 
of lysine analogue use. Information about non-responders was not available to assess for potential biases, although the possibility of participation bias might be present, given that the surgeons who chose not to respond might not have been familiar with lysine analogues.

\section{CONCLUSIONS}

Our survey found that lysine analogues are infrequently used in oncologic surgical procedures at our institution and that the timing of administration often varied. Many oncologic surgeons at our institution are unfamiliar with the benefits and side effects of lysine analogues and preferentially use topical hemostatic agents. Our results demonstrate that future trials exploring the efficacy and safety of lysine analogues in the oncology population are needed.

\section{CONFLICT OF INTEREST DISCLOSURES}

We have read and understood Current Oncology's policy on disclosing conflicts of interest, and we declare that we have none.

\section{AUTHOR AFFILIATIONS}

${ }^{*}$ Clinical Epidemiology Program, Centre for Practice Changing Research, The Ottawa Hospital Research Institute, †University of Ottawa, School of Epidemiology and Public Health, and ${ }^{\ddagger}$ Division of Urology, Department of Surgery, The Ottawa Hospital, Ottawa, ON.

\section{REFERENCES}

1. Karkouti K, Dattilo KM. Perioperative hemostasis and thrombosis. Can J Anaesth 2006;53:1260-2.

2. Marietta M, Facchini L, Pedrazzi P, Busani S, Torelli G. Pathophysiology of bleeding in surgery. Transplant Proc 2006;38:812-14.

3. Alter HJ, Klein HG. The hazards of blood transfusion in historical perspective. Blood 2008;112:2617-26.

4. Mannucci PM, Levi M. Prevention and treatment of major blood loss. N Engl J Med 2007;356:2301-11.

5. Breau RH, Kokolo MB, Punjani N, et al. The effects of lysine analogs during pelvic surgery: a systematic review and meta-analysis. Transfus Med Rev 2014;28:145-55.

6. Hutton B, Joseph L, Fergusson D, Mazer CD, Shapiro S, Tinmouth A. Risks of harms using antifibrinolytics in cardiac surgery: systematic review and network meta-analysis of randomised and observational studies. BMJ2012;345:e5798.

7. Ker K, Edwards P, Perel P, Shakur H, Roberts I. Effect of tranexamic acid on surgical bleeding: systematic review and cumulative meta-analysis. BMJ 2012;344:e3054.

8. Vaněk T, Straka Z. Topical use of tranexamic acid in cardiac surgery-a review and meta-analysis of four randomized controlled trials. Cor et Vasa 2013;55:e184-9.

9. Farrow LS, Smith TO, Ashcroft GP, Myint PK. A systematic review of tranexamic acid in hip fracture surgery. $\mathrm{Br} \mathrm{J}$ Clin Pharmacol 2016;82:1458-70.

10. Lin ZX, Woolf SK. Safety, efficacy, and cost-effectiveness of tranexamic acid in orthopedic surgery. Orthopedics 2016;39:119-30.

11. Molenaar IQ, Warnaar N, Groen H, Tenvergert EM, Slooff MJH, Porte RJ. Efficacy and safety of antifibrinolytic drugs in liver transplantation: a systematic review and meta-analysis. $\mathrm{Am}$ J Transplant 2007;7:185-94.

12. Kagoma YK, Crowther MA, Douketis J, Bhandari M, Eikelboom J, Lim W. Use of antifibrinolytic therapy to reduce transfusion in patients undergoing orthopedic surgery: a systematic review of randomized trials. Thromb Res 2009; 123:687-96.

13. Li ZJ, Fu X, Xing D, Zhang HF, Zang JC, Ma XL. Is tranexamic acid effective and safe in spinal surgery? A meta-analysis of randomized controlled trials. Eur Spine J 2013;22:1950-7.

14. Basta MN, Stricker PA, Taylor JA. A systematic review of the use of antifibrinolytic agents in pediatric surgery and implications for craniofacial use. Pediatr Surg Int 2012;28:1059-69.

15. Olsen JJ, Skov J, Ingerslev J, Thorn JJ, Pinholt EM. Prevention of bleeding in orthognathic surgery-a systematic review and meta-analysis of randomized controlled trials. J Oral Maxillofac Surg 2016;74:139-50.

16. Zehtabchi S, Abdel BakiSG, Falzon L, Nishijima DK. Tranexamic acid for traumatic brain injury: a systematic review and meta-analysis. Am J Emerg Med 2014;32:1503-9.

17. Punjani N, Lavallee LT, Momoli F, et al. Blood transfusion and hemostatic agents used during radical cystectomy. Can Urol Assoc J 2013;7:E275-80.

18. Truong JL, Cyr DP, Lam-McCulloch J, Cleary SP, Karanicolas PJ. Consensus and controversy in hepatic surgery: a survey of Canadian surgeons. J Surg Oncol 2014;110:947-51.

19. Montroy J, Fergusson NA, Hutton B, et al. The safety and efficacy of lysine analogues in cancer patients: a systematic review and meta-analysis. Transfus Med Rev 2017;31:141-8.

20. Pereira J, Phan T. Management of bleeding in patients with advanced cancer. Oncologist 2004;9:561-70.

21. McAlpine K, Breau RH, Knee C, et al. Venous thromboembolism and transfusion after major abdominopelvic surgery. Surgery 2019;166:1084-91.

22. Dillman DA, Smyth JD. Design effects in the transition to Web-based surveys. Am J Prev Med 2007;32(suppl):S90-6.

23. Graham ID, Fergusson D, McAuley, Laupacis A. The use of technologies to minimize exposure to perioperative allogeneic blood transfusion in elective surgery. A survey of Canadian hospitals. Int J Technol Assess Health Care 2000;16:228-41.

24. Baron JA, Gridley G, Weiderpass E, Nyren O, Linet M. Venous thromboembolism and cancer. Lancet 1998;351:1077-80.

25. Lee AY, Levine MN. Venous thromboembolism and cancer: risks and outcomes. Circulation 2003;107(suppl 1):I17-21.

26. Fergusson D, Blair A, Henry D, et al. Technologies to minimize blood transfusion in cardiac and orthopedic surgery. Results of a practice variation survey in nine countries. International Study of Peri-operative Transfusion (ISPOT) investigators. Int J Technol Assess Health Care 1999;15:717-28.

27. Seyednejad H, Imani M, Jamieson T, Seifalian AM. Topical haemostatic agents. BrJ Surg 2008;95:1197-225.

28. Esper SA, Waters JH. Intra-operative cell salvage: a fresh look at the indications and contraindications. Blood Transfus 2011; 9:139-47.

29. Froessler B, Palm P, Weber I, Hodyl NA, Singh R, Murphy EM. The important role for intravenous iron in perioperative patient blood management in major abdominal surgery: a randomized controlled trial. Ann Surg 2018;267:e39-40.

30. Holst LB, Petersen MW, Haase N, Perner A, Wetterslev J. Restrictive versus liberal transfusion strategy for red blood cell transfusion: systematic review of randomised trials with metaanalysis and trial sequential analysis. BMJ 2015;350:h1354.

31. Zhou X, Zhang C, Wang Y, Yu L, Yan M. Preoperative acute normovolemic hemodilution for minimizing allogeneic blood transfusion: a meta-analysis. Anesth Analg 2015;121:1443-55.

32. Litton E, Xiao J, Ho KM. Safety and efficacy of intravenous iron therapy in reducing requirement for allogeneic blood transfusion: systematic review and meta-analysis of randomised clinical trials. BMJ 2013;347:f4822.

33. Foltys D, Zimmermann T, Heise M, et al. Liver transplantation for hepatocellular carcinoma-is there a risk of recurrence caused by intraoperative blood salvage autotransfusion? Eur Surg Res 2011;47:182-7.

34. Futamura N, Nakanishi H, Hirose H, Nakamura S, Tatematsu $M$. The effect of storage on the survival of cancer cells in blood and efficient elimination of contaminating cancer cells by a leukocyte depletion filter. Am Surg 2005;71:585-90. 